\title{
CRACK ANALYSIS IN BIMATERIAL INTERFACES USING T-SPLINE BASED XIGA
}

\author{
Sadam Houcine Habib, Idir Belaidi \\ University of M'hamed Bougara, Department of Mechanical Engineering, Boumerdes, Algeria \\ e-mail: hb_houcine@yahoo.fr; idir.belaidi@gmail.com
}

\begin{abstract}
Analysis-suitable T-splines are used for the modeling and analyzing of cracks in bimaterial interfaces within the framework of an extended isogeometric analysis (XIGA). The crack tip enrichment functions of bimaterial interface cracks are implemented to reproduce singular fields, and the signed distance functions are used to treat the crack face and the interface in the models. A compatible local refinement algorithm is applied to refine location of the crack and the interface, which helps one to avoid produce excessive propagation of control points. The mixed mode stress intensity factors (SIFs) which are evaluated by the interaction integral (M-integral) are used as analysis parameters. Numerical simulations are performed to analyze the problem and to examine the efficiency of the proposed method. The obtained results are compared with other available results.
\end{abstract}

Keywords: extended isogeometric analysis, T-splines, bimaterial interface cracks, enrichment functions, local refinement

\section{Introduction}

As its name indicates, a composite material is made up of two or more different constituents; it has properties that cannot be obtained together by one of the individual constituents, such as high specific strength and stiffness, good durability and good corrosion resistance. Due to their properties, composites have been developed and used in various industrial and engineering applications, like those in aerospace, aircraft, automotive industries, etc. However, these materials are not immune to manufacture defects especially from those which are created as interfacial cracks. This problem greatly influences the behavior of structures and can cause brutal fracture.

The mechanical behavior of composite materials needs more understanding, especially in the presence of strong and weak discontinuities. Many analytical studies were performed based upon the work of Williams (1959) for understanding the problem of bimaterial interface cracks, such as (Erdogan 1963; Rice and Sih, 1965; Sun and Jih, 1987; Hutchinson et al., 1987; Rice, 1988; Evans et al., 1990). However, the complexity of analytical solutions even for simple cases requires the modelling of mechanical behavior of this problem using effective numerical methods. Several investigations have been developed in this domain, via the boundary element method (BEM) (Lee and Choi, 1988; Yuuki and Xu, 1994; Miyazaki et al., 1993), finite element method (FEM) (Ikeda et al., 2006), element free Galerkin method (EFGM) (Pant et al., 2011), extended finite element method (XFEM) (Nagashima et al., 2003; Liu et al., 2004; Belytschko and Gracie, 2007) and other methods (Zhou et al., 2013, 2014; An et al., 2013). Recently, a large field was opened by Hughes et al. (2005) offering the possibility of introducing computer aided design (CAD) tools in the analysis methods using the isoparametric concept. The basic idea of this novel alternative method, called isogeometric analysis (IGA), is to exploit the technologies of computational geometry as shape bases to describe the geometry exactly, also for the approximation of unknown fields. Following this discovery, several researches in various 
fields have been conducted by this method, including: fluid-structure interaction (Bazilevs et al., 2006), composite materials (Peković et al., 2015), elastic-plastic analysis (Kalali et al., 2016), electromagnetic problems (Buffa et al., 2010), turbulent flow (Bazilevs and Akkerman, 2010), contact problems (Temizer et al., 2011), aero-dynamics (Hsu et al., 2011), heat transfer (Anders et al., 2012) and fluid mechanics (Evan and Hudhes, 2013). For more details about IGA, a recent review has been published, see Nguyen et al. (2015). In fracture mechanics problems, IGA has been also applied in different studies (Verhoosel et al., 2011; Borden et al., 2012; Nguyen and Nguen-Xuan, 2013; Nguyen et al., 2014; Peng et al., 2014), however Benson et al. (2010) and De Luycker et al. (2011) proposed extended isogeometric analysis (XIGA) for modelling cracks. In this method the general principle of the XFEM is used in IGA by including the asymptotic and signed distance enrichment functions. Therefore, this method has the advantages of both XFEM and IGA, which are summarized by the ability to represent complex geometries independently of any discontinuities and without explicit meshing to obtain solutions with higher orders. Some applications in fracture mechanics have been checked by the XIGA, such as in the cases of homogeneous materials (Ghorashi et al., 2012; Bhardwaj and Singh, 2015), functionally graded material (Bhardwaj et al., 2015a,c) and bimaterial interfaces (Bhardwaj et al., 2015b; Jia et al., 2015), where the non-uniform rational B-splines (NURBS) are used. Also, orthotropic media have been studied using T-spline based XIGA (Ghorashi et al., 2015).

There are many CAD basis functions that can be used in IGA, where the Non Uniform Rational B-splines (NURBS) are widely used due to their properties, like continuity, smoothness, variation diminishing, convex hull and possibility of using knot insertion and degree elevation refinements. They have the ability to describe exactly all conic sections but they have difficulties in certain complex geometries which cannot be avoided even by using multiple patches, where NURBS generate a complicated mesh which leads to produce superfluous control points. In order to handle these disadvantages, Sederberg et al. (2003) proposed a T-spline as a generalized tool of NURBS, in which the index space (T-mesh) locally refined using T-junctions (Sederberg et al., 2004). Therefore, the major advantages of this technique are the local refinement and the ability to represent complex geometries with a minimal number of control points compared with those used in NURBS.

According to their ability in engineering design, T-splines have been used by analysis to serve as basis functions for IGA in many advanced searches. However T-spline bases are not always valid to be used in analysis for different geometric configurations, because the linear independence and partition unity properties are not always ensured. Li et al. (2012) introduced analysis-suitable T-splines, where for any choice of knot vectors the blending functions are linearly independent. Like NURBS bases, analysis-suitable T-spline bases have the properties of the analysis basis functions. Moreover, they provide an efficient algorithm which allows making highly localized refinement (Scott et al., 2012).

In this paper, the interface crack in the case of $2 \mathrm{D}$ composites is analyzed using $\mathrm{T}$-spline based XIGA; the accuracy of this approach is first tested in isotropic materials. The analysis-suitable T-spline and its refinement algorithm are highlighted.

\section{Analysis-suitable T-splines}

An analysis-suitable T-spline is founded when the T-mesh (T-mesh is a mesh of rectangular elements that is defined by the lines corresponding to knot values of the parametric vectors) provides a restricted topology that has no intersecting T-junction extensions. The T-junction extension is defined in each T-junction vertex by an interval which includes two distances. The first distance is between the T-junction and the two next adjoining edges or vertices in the direction of missing edge, while the second distance is between the T-junction and one edge 
or vertex in the other direction, as shown in Fig. 1b. The T-mesh that shows all T-junction extensions can be called extended T-mesh. An empty extended T-mesh means there are no intersections between T-junction extensions (see Fig. 1c), which means the T-mesh is analysis-suitable.

(a)

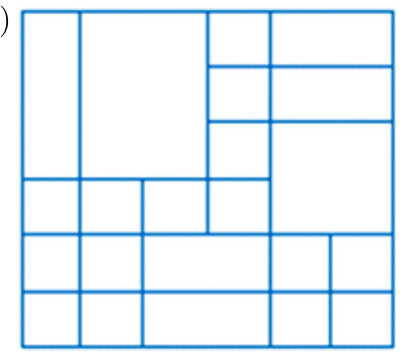

(b)

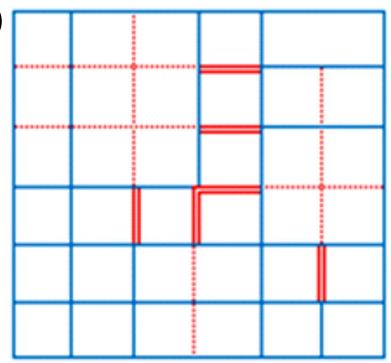

(c)

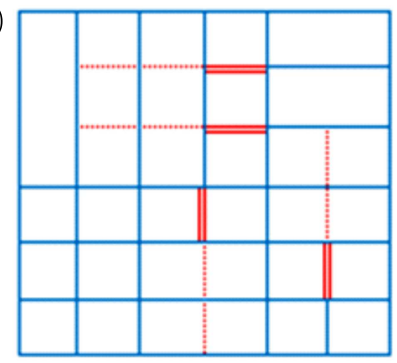

Fig. 1. An example depicts: (a) T-mesh, (b) extended T-mesh and (c) empty extended T-mesh

In order to make local refinement of analysis-suitable T-spline spaces, Scott et al. (2012) introduced an algorithm consisting of the following steps:

- create the refined T-mesh $T_{2}$ from the original analysis-suitable T-mesh $T_{s 1}$,

- form the extended T-mesh of $T_{2}$.

- if the extended T-mesh of $T_{2}$ has intersecting T-junction extensions, one edge must be inserted into $T_{2}$ in such a way that reduces the number of the intersections,

- repeat step 3 until the extended T-mesh has no intersecting T-junction extensions,

- compute the refinement matrix $\mathbf{M}$.

For more details, see (Scott et al., 2012).

\section{Extended isogeometric analysis (XIGA)}

XIGA (Benson et al., 2010; De Luycker et al., 2011) uses the same methodology of the extended finite element method (XFEM) for the modelling of discontinuities but with basis functions derived from geometry like in isogeometric analysis (Hughes et al., 2005). For crack problems, XIGA provides the possibility of modelling the crack independently of the mesh and within exactly presented geometry. Uncommonly, in this study, T-splines are adopted in XIGA using analysis-suitable T-splines to approximate the displacement in any point $\zeta=(\xi, \eta)$ as follows

$$
\mathbf{u}(\zeta)=\sum_{i=1}^{n_{s}} \mathbf{R}_{i}(\zeta) \mathbf{u}_{i}+\sum_{j=1}^{n_{c f}} \mathbf{R}_{j}(\zeta) H(\zeta) \mathbf{a}_{j}+\sum_{k=1}^{n_{c t}} \mathbf{R}_{k}(\zeta)\left(\sum_{\ell=1}^{4} F_{\ell}(\zeta) \mathbf{b}_{k}^{\ell}\right)+\sum_{t=1}^{n_{i}} \mathbf{R}_{t}(\zeta) \chi(\zeta) \mathbf{c}_{t}
$$

where $\mathbf{R}$ is the T-spline basis function extracted from an empty extended T-mesh, $H$ is the Heaviside function used for the modelling of the crack face, it takes value 1 above the crack and -1 below the crack, $F$ are the crack-tip enrichment functions, $\mathbf{u}_{i}, \mathbf{a}_{j}, \mathbf{b}_{k}$ and $\mathbf{c}_{t}$ are the displacement vectors corresponding to $n_{s}, n_{c f}, n_{c t}$ and $n_{t}$ control points, respectively. The fourth term is used when there is no coincidence between the interface and the finite element mesh for the modelling of weak discontinuity. The enrichment function of Moës et al. (2003) $\chi$ can be used

$$
\chi(\zeta)=\sum \mathbf{R}_{I}(\zeta)\left|\phi_{I}\right|-\left|\sum \mathbf{R}_{I}(\zeta) \phi_{I}\right|
$$

where $\phi$ is the signed distance value of the interface control points. 
The enrichment functions of bimaterial interface cracks were derived by Sukumar et al. (2004) as

$$
\begin{aligned}
& \left\{F_{\ell}(r, \theta)\right\}_{\ell=1}^{12}=\left\{\sqrt{r} \cos (\varepsilon \log r) \mathrm{e}^{-\varepsilon \theta} \sin \frac{\theta}{2}, \sqrt{r} \cos (\varepsilon \log r) \mathrm{e}^{-\varepsilon \theta} \cos \frac{\theta}{2}, \sqrt{r} \cos (\varepsilon \log r) \mathrm{e}^{\varepsilon \theta} \sin \frac{\theta}{2},\right. \\
& \quad \sqrt{r} \cos (\varepsilon \log r) \mathrm{e}^{\varepsilon \theta} \cos \frac{\theta}{2}, \sqrt{r} \cos (\varepsilon \log r) \mathrm{e}^{\varepsilon \theta} \sin \frac{\theta}{2} \sin \theta, \sqrt{r} \cos (\varepsilon \log r) \mathrm{e}^{\varepsilon \theta} \cos \frac{\theta}{2} \sin \theta \\
& \quad \sqrt{r} \sin (\varepsilon \log r) \mathrm{e}^{-\varepsilon \theta} \sin \frac{\theta}{2}, \sqrt{r} \sin (\varepsilon \log r) \mathrm{e}^{-\varepsilon \theta} \cos \frac{\theta}{2}, \sqrt{r} \sin (\varepsilon \log r) \mathrm{e}^{\varepsilon \theta} \sin \frac{\theta}{2}, \\
& \left.\quad \sqrt{r} \sin (\varepsilon \log r) \mathrm{e}^{\varepsilon \theta} \cos \frac{\theta}{2}, \sqrt{r} \sin (\varepsilon \log r) \mathrm{e}^{\varepsilon \theta} \sin \frac{\theta}{2} \sin \theta, \sqrt{r} \sin (\varepsilon \log r) \mathrm{e}^{\varepsilon \theta} \cos \frac{\theta}{2} \sin \theta\right\}
\end{aligned}
$$

\section{Numerical simulations}

Here, the analysis-suitable T-spline is used in XIGA to simulate the crack in homogeneous isotropic and bimaterial interfaces. Two numerical examples are considered for each material type in plane static problems, where mode I and mode II SIFs are evaluated and compared with other numerical and analytical results. First, the isotropic material is considered in a rectangular plate with an edge crack in order to study the convergence and the domain independence in the computations of SIF, also an isotropic square plate with a center crack is analyzed for different crack angles to verify the accuracy of the proposed approach. Then, numerical applications in the form of parametric studies are considered for edge and center interface cracks in finite rectangular plates.

In all geometric models (NURBS and T-splines) the cubic order is used in both parametric directions, where the weights are taken as unity. In the edge crack problems, the geometry is refined locally once, while for the center crack problems the geometry is refined locally twice. Four types of finite elements are distinguished in these examples according to their positions with respect to the crack, the standard element contains $3 \times 3$ Gauss points. The element having tip enriched control points contains $7 \times 7$ Gauss points and the sub-triangle technique (Ghorashi et al., 2011) is used for the tip-element by 13 Gauss points in each triangle, however the split element contains $6 \times 6$ Gauss points for the horizontal crack problems and the sub-triangle technique is used by 13 Gauss points in each triangle for the inclined crack problems. The SIFs are evaluated using interaction integral (Yau and Wang, 1984), wherein the crack tip element is not considered in the calculation.

\subsection{Homogeneous isotropic material}

In this case, we simulate a finite rectangular plate containing an edge crack (Fig. 2a) and a square plate containing an inclined central crack (Fig. 2b), subjected to unit uniaxial tension in plane stress state. The convergence of the proposed approach is studied for the edge crack problem with normalized M-integral radius equal to 1 using five different control net configurations (200, 296, 362, 754 and 1800 control points), all shown in Fig. 3. The errors of the normalized SIF values obtained from the proposed approach which are shown in Table. 1 are computed using the following equation

$$
\bar{K}_{I}=\frac{K_{I}}{\sigma \sqrt{\pi a}}=T_{I}\left(\frac{a}{L}\right)
$$

where $T_{I}(a / L)$ is the analytical formula which corresponds to mode I, it can be computed as (Tada et al., 2000)

$$
T_{I}=1.122-0.231 \frac{a}{L}+10.55\left(\frac{a}{L}\right)^{2}-21.71\left(\frac{a}{L}\right)^{3}+30.382\left(\frac{a}{L}\right)^{4}
$$


(a)

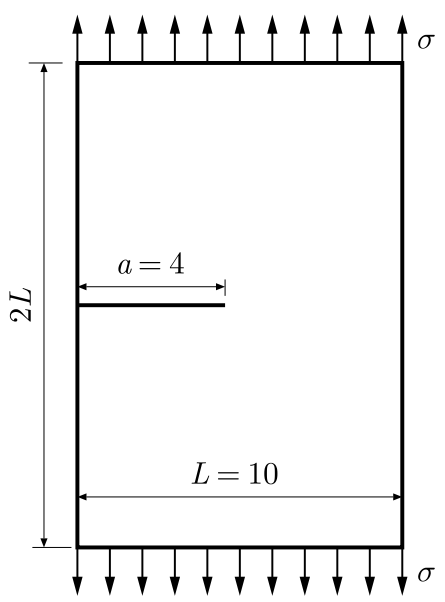

(b)

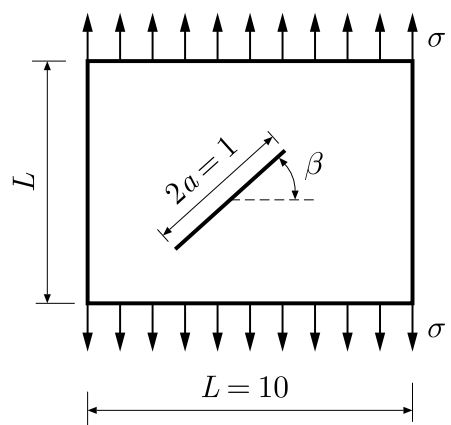

Fig. 2. Geometries and loading of the homogeneous isotropic examples (a) rectangular plate with an edge crack and (b) square plate with a center inclined crack

(a) 0000000000 0000000000 0000000000

0000000000

0000000000

0000000000

000000000

0000000000 0000000000

0000000000

0000000000

0000000000

0000000000

000000000

0000000000

0000000000

0000000000

0000000000

000000000

(b)

) 0000000000 0000000000 0000000000 000000000 0000000000 0000000000 0000000000 0000000000 - $8900 \times 79$ - 888 理8

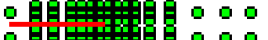

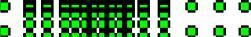
000000000 00000000 - 0000 0.000000 00000000 0000000000 (c) 000000000000( 000000000000 000000000000 000000000000 000000000000 000000000 00000000 00000000 o

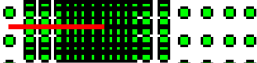
0 00000000 00000000000 - 0 o a o o -

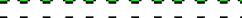
000000000000 000000000000 000000000000

) 90989990909998 (e) 00000000000000 09090909090909 00000000009090 00000000000000 gogggggoggogg 000000000 ogogogoggogogo gent ! ! ! ! ! !

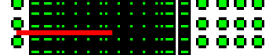
ga:.. : : : : : : : : : :

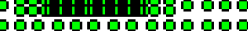
goggogggoggggo 00000000000000 09090909090909 000000000000 gogogogogogogo

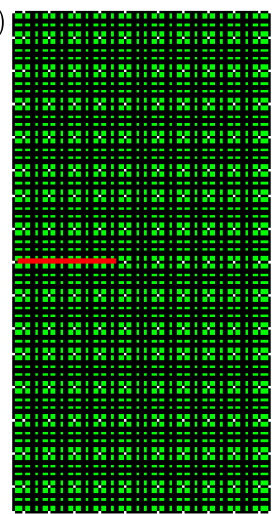

Fig. 3. Different mesh configurations used in the convergence study: (a) 200 points, (b) 296 points, (c) 362 points, (d) 788 points and (e) 1800 points

Table 1. Convergence of the SIF for various control nets

\begin{tabular}{|c|c|c|}
\hline Control points & $\bar{K}_{I}$ & Error [\%] \\
\hline \hline 200 & 2.1275 & 1.0257 \\
\hline 296 & 2.1189 & 0.6173 \\
\hline 362 & 2.1121 & 0.2944 \\
\hline 754 & 2.1098 & 0.1852 \\
\hline 1800 & 2.1131 & 0.3419 \\
\hline
\end{tabular}

The first and the last meshes in Fig. 3 represent a special case of T-splines which is NURBS. According to Table 1, analysis suitable T-splines give us precise results for a different number of control points (meshes 2, 3 and 4), even for the minimal number of control points compared to NURBS (mesh 4 compared to mesh 5) and that attributed to the local refinement property. Table 2 compares the results of the normalized SIF for different radius to study the domain independence in T-spline meshes. We observe that the SIF values are almost not sensitive to the radius of the M-integral. The contour plots of the normal stress component $\sigma_{y y}$ and the vertical displacement $u_{y}$ are illustrated in Fig. 4.

For the square plate, we used a mesh consisting of 788 control points and 689 elements (Figs. 5a and 5b) to evaluate the normalized mixed mode SIF for $a=0.5$ in different inclined angles. The exact SIFs of this problem can be obtained by the following equations

$$
K_{I}=\sigma_{0} \sqrt{\pi a} \cos ^{2} \beta \quad K_{I I}=\sigma_{0} \sqrt{\pi a} \sin \beta \cos \beta
$$


Table 2. Domain independence study

\begin{tabular}{|c|c|c|c|c|c|c|}
\hline \multirow{2}{*}{ Radius } & \multicolumn{2}{|c|}{ Mesh 2 } & \multicolumn{2}{c|}{ Mesh 3 } & \multicolumn{2}{c|}{ Mesh 4 } \\
\cline { 2 - 7 } & $K_{I}$ & Error [\%] & $K_{I}$ & Error [\%] & $K_{I}$ & Error [\%] \\
\hline \hline 0.6 & 2.1131 & 0.3419 & 2.1280 & 1.0494 & 2.1245 & 0.8832 \\
\hline 0.7 & 2.1256 & 0.9355 & 2.1202 & 0.6790 & 2.1142 & 0.3941 \\
\hline 0.8 & 2.1256 & 0.9355 & 2.1209 & 0.7123 & 2.1122 & 0.2992 \\
\hline 0.9 & 2.1218 & 0.7550 & 2.1177 & 0.5603 & 2.1122 & 0.2992 \\
\hline 1.0 & 2.1189 & 0.6173 & 2.1121 & 0.2944 & 2.1098 & 0.1852 \\
\hline 1.1 & 2.1146 & 0.4131 & 2.1118 & 0.2802 & 2.1109 & 0.2374 \\
\hline
\end{tabular}

(a)

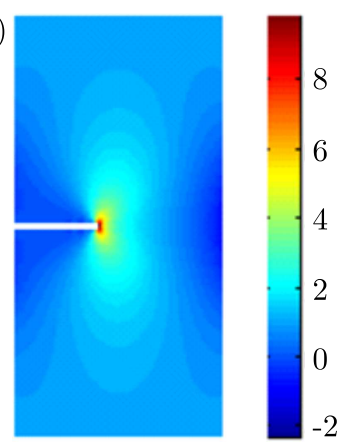

(b)

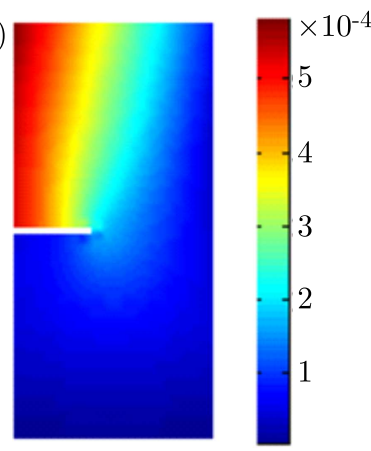

Fig. 4. Graphical visualization: (a) normal stress and (b) vertical displacement

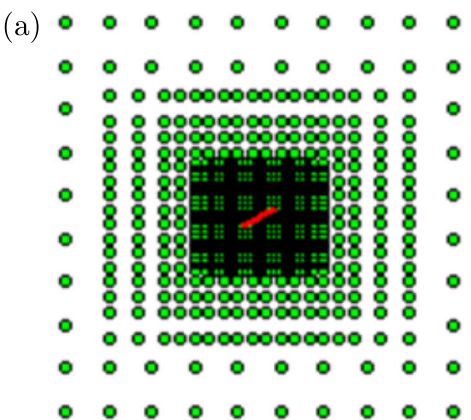

(b)

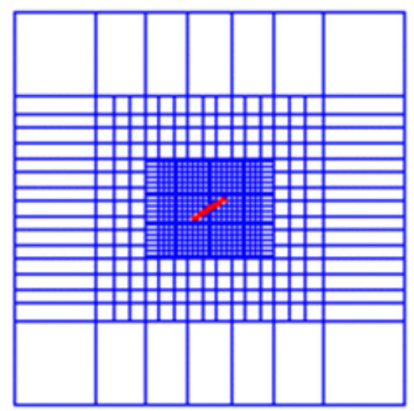

(c)

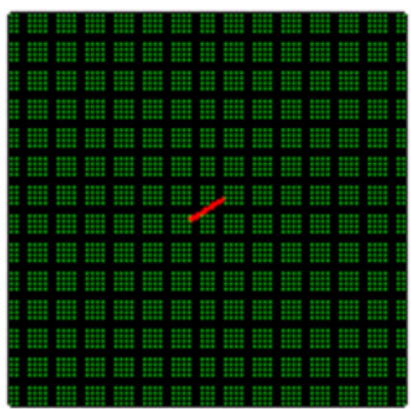

Fig. 5. The meshes used for the isotropic square plate: (a) T-spline control net (788 points), (b) elements corresponding to the T-spline control net (689 elements) and (c) NURBS control net (4625 points)
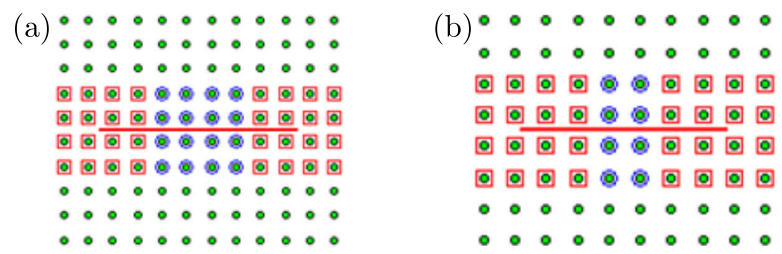

Fig. 6. The crack tip (red squares) and the crack face (blue circles) enriched points of: (a) T-spline control net and (b) NURBS control net, in the case $\beta=0$

Figure 6a depicts the enriched control points that correspond to the crack face and crack tip elements. Figure 7 shows a comparison between the normalized SIFs calculated by the proposed approach and those derived from the exact solution and NURBS-based XIGA. A uniform NURBS mesh is used Fig. 6c, its enriched control points are presented in Fig. 6b. As seen in both modes, there is a very close agreement between the T-spline results and the other results. 
(a)

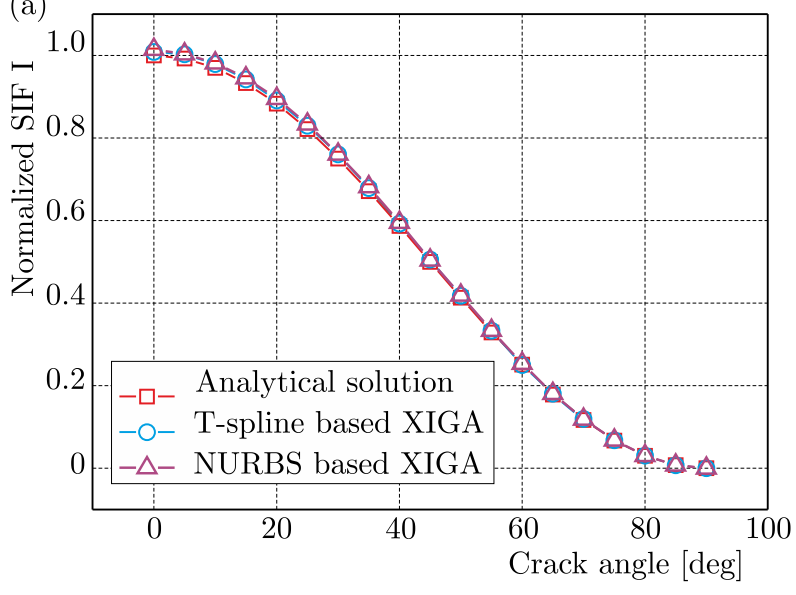

(b)

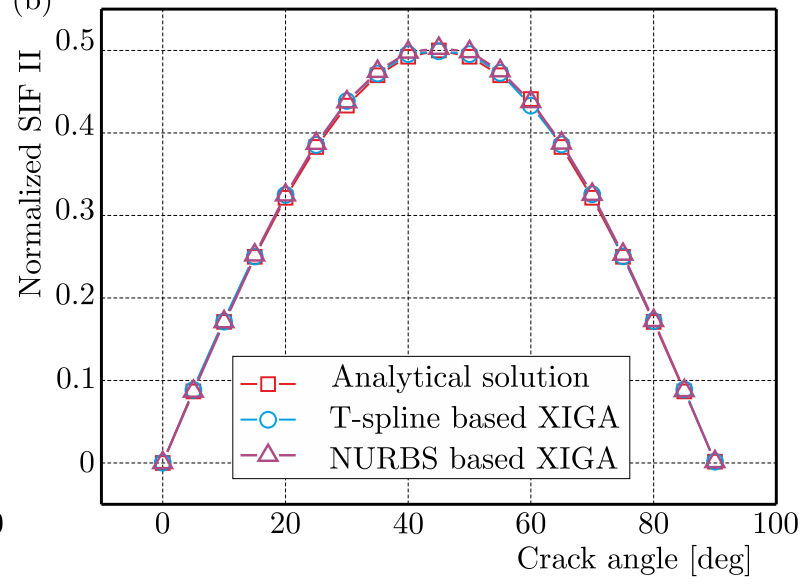

Fig. 7. Variations of normalized mode I and II SIFs with respect to different crack angles using the analysis-suitable T-splines, NURBS and exact solution for the square plate problem

(a)

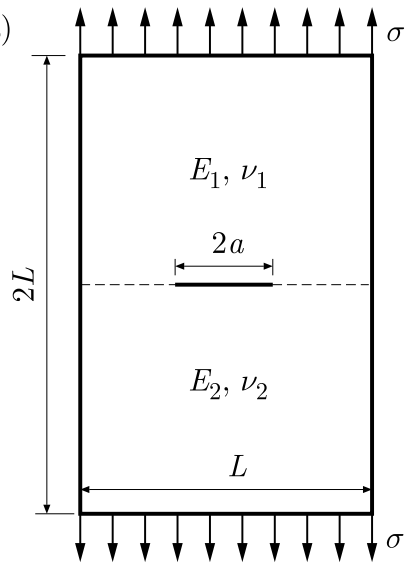

(b)

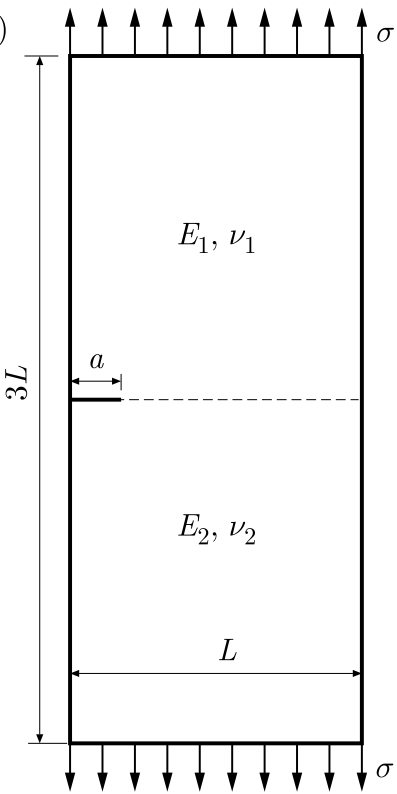

Fig. 8. Geometries and loading of the bimaterial interface examples: (a) interface center crack and (b) interface edge crack

\subsection{Bimaterial interface crack}

We consider two finite rectangular plates subjected to uniaxial tensions in plane stress conditions, each one constituted of two dissimilar materials and cracked in the interface as shown in Fig. 8. Different ratios of Young's modulus $\left(E_{1} / E_{2}=2,3,4,10\right.$ and 100) with fixed Poisson ratios $\left(\nu_{1}=\nu_{2}=0.3\right)$ are taken in the simulation. Similar problems were solved before by Miyazaki et al. (1993) utilizing the boundary element method (BEM), Nagashima et al. (2003) utilizing an extended finite element method (XFEM), Matsumto et al. (2000) making use of the interaction energy release rates and BEM sensitivity and Liu et al. 2004) using XFEM for direct evaluation of the mixed mode SIF. For the center crack problem (Fig. 8a), we use a mesh consisting of 3132 control points and 2925 elements as shown in Figs. 9a and 9b. For the edge crack problem (Fig. 8b), we use a mesh consisting of 1446 control points and 1235 elements as shown in Figs. 9c and 9d. The enriched control points are defined in Fig. 10. In order to verify the accuracy of the obtained results, the normalized SIFs are compared with those obtained by 
other methods in Fig. 11 for the center crack problem $(2 a / L=0.4)$ and in Fig. 12 for the edge crack problem $(a / L=0.3)$. Figures 13 and 14 illustrate variations of the normalized SIFs in terms of crack lengths for the center and edge crack problems, respectively. For more details, check Table 3 and Table 4.
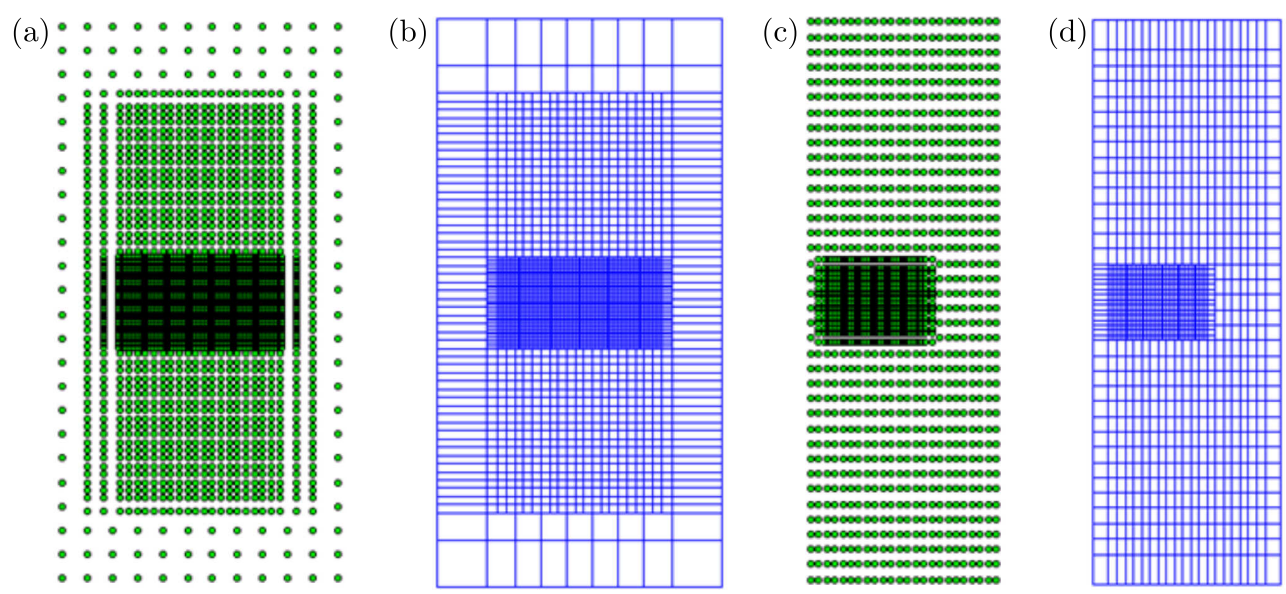

Fig. 9. T-spline meshes used for the bimaterial interface examples: (a) control net for the interface center crack (793 points), (b) mesh for the interface center crack (688 elements), (c) control net for the interface edge crack (566 points) and (d) mesh for the interface edge crack (431 elements)

(a)

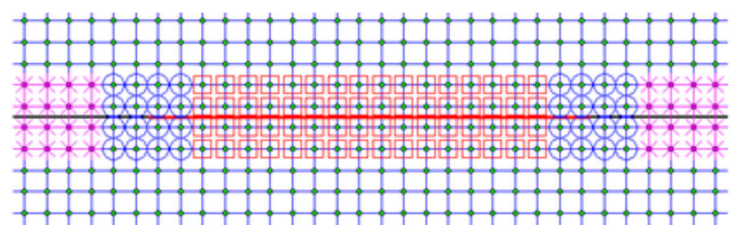

(b)

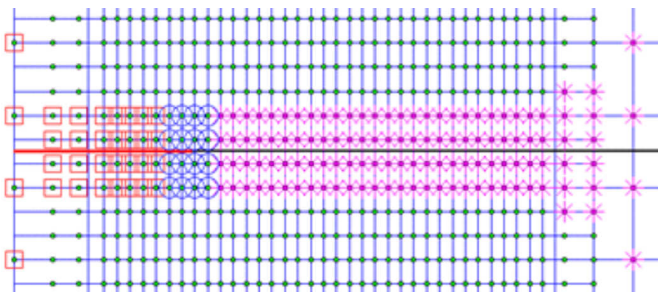

Fig. 10. Crack tip, crack face and interface enriched control points: (a) interface center crack and (b) interface edge crack
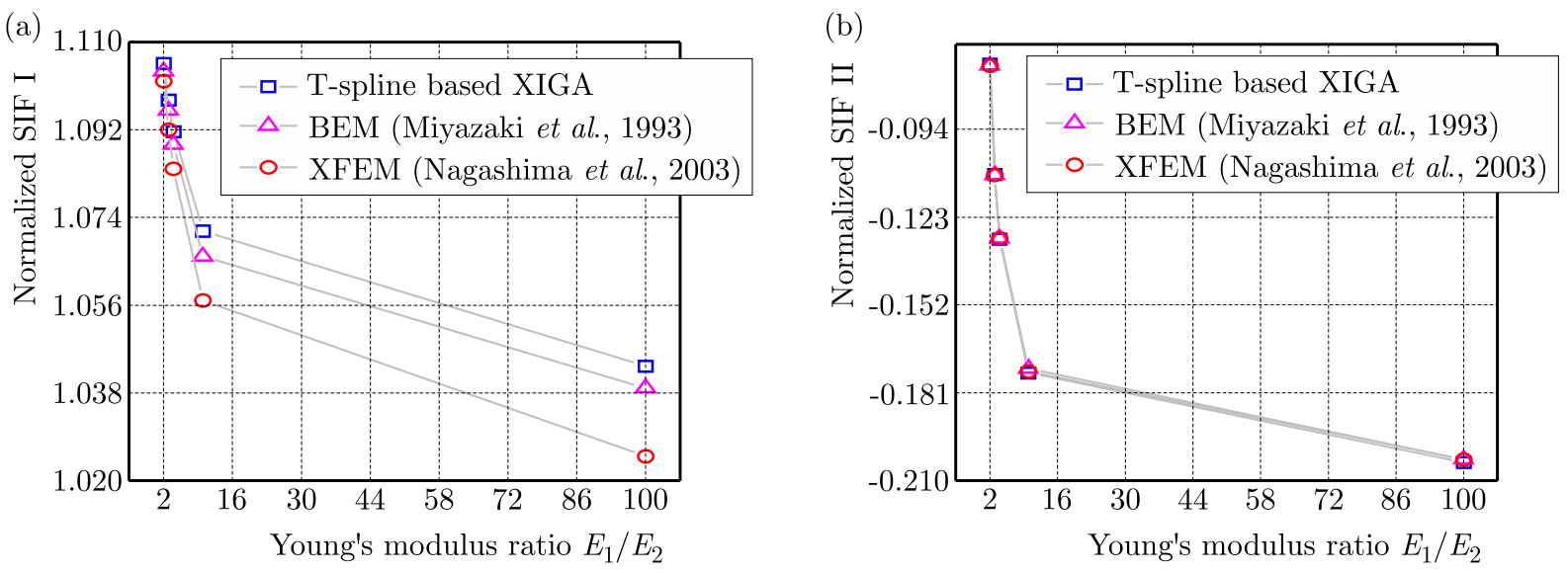

Fig. 11. Variations of normalized mode I and II SIFs with respect to different Young's modulus ratios using T-spline based XIGA, BEM and XFEM for the center interface crack $(2 a / L=0.4)$ 

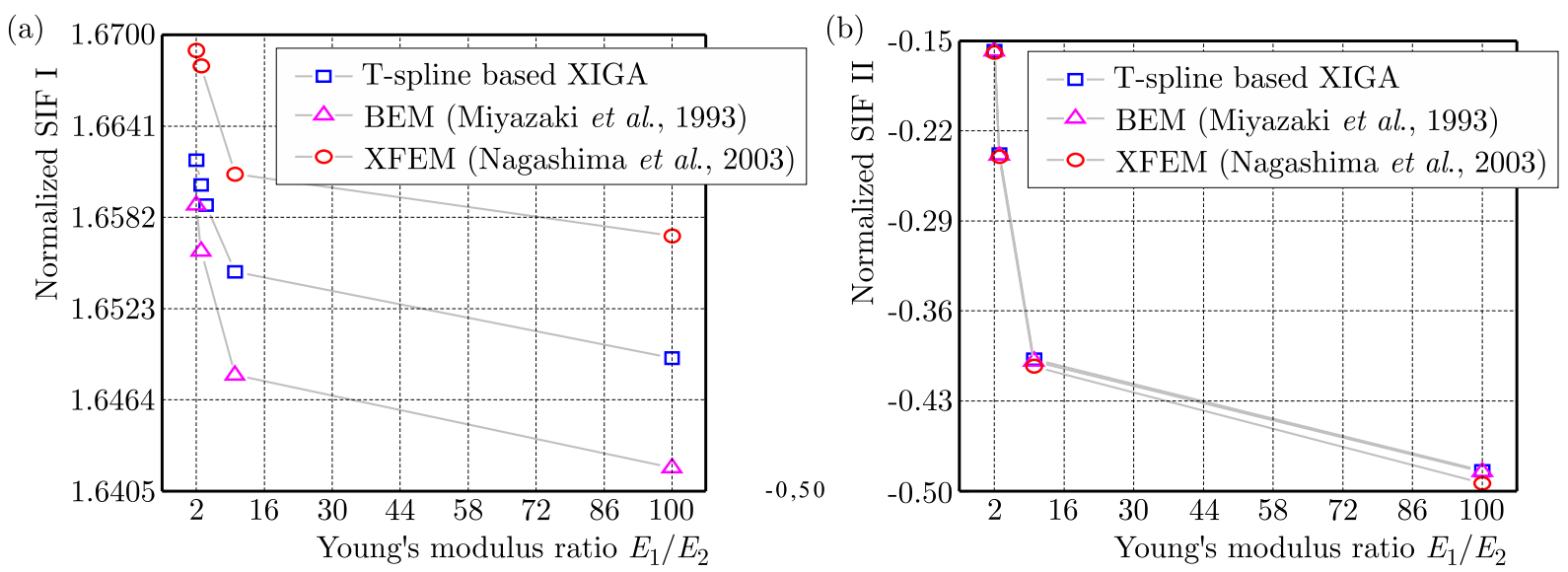

Fig. 12. Variations of normalized mode I and II SIFs with respect to different Young's modulus ratios using T-spline based XIGA, BEM and XFEM for the edge interface crack $(a / L=0.3)$
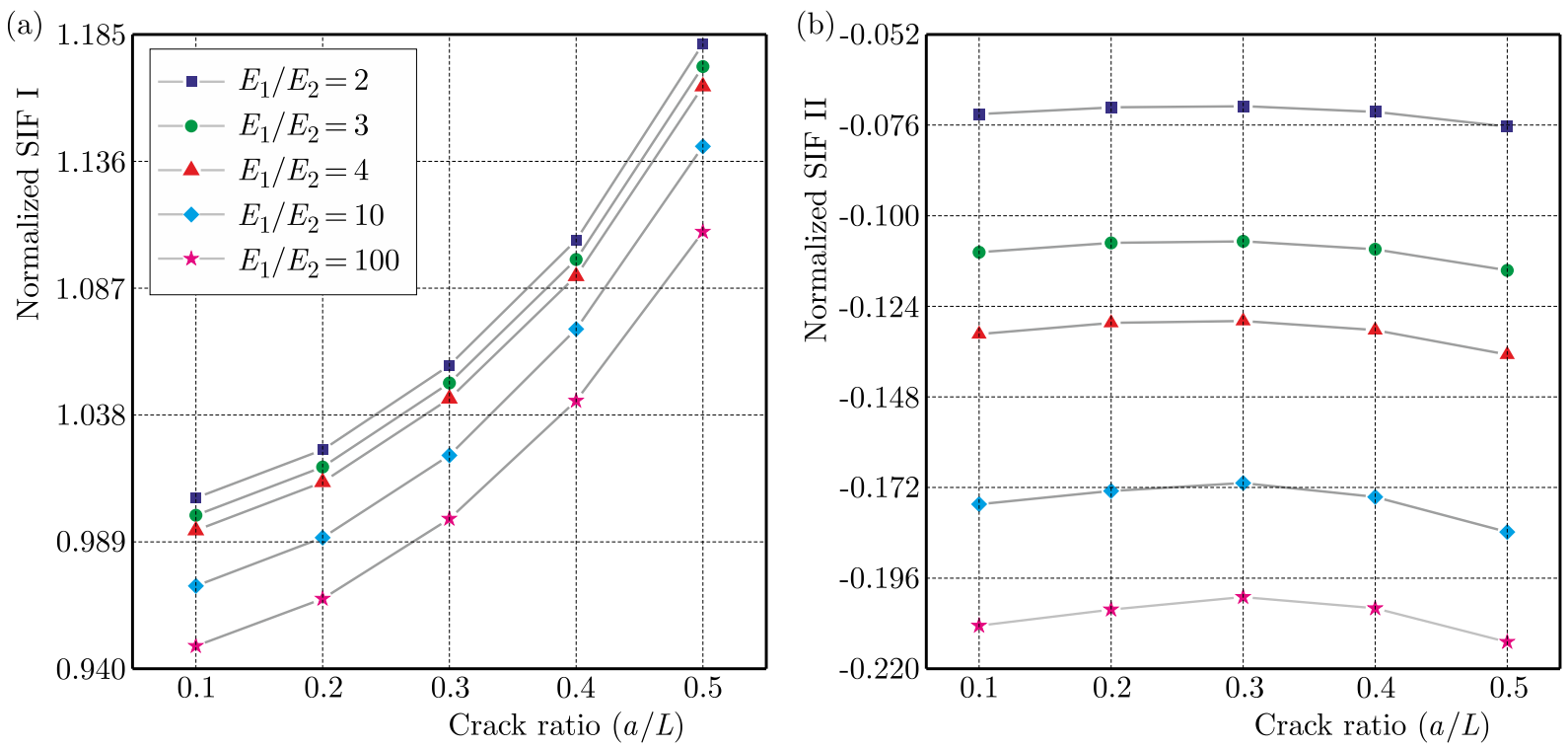

Fig. 13. The effect of Young's modulus ratio on the normalized SIFs for the center interface crack
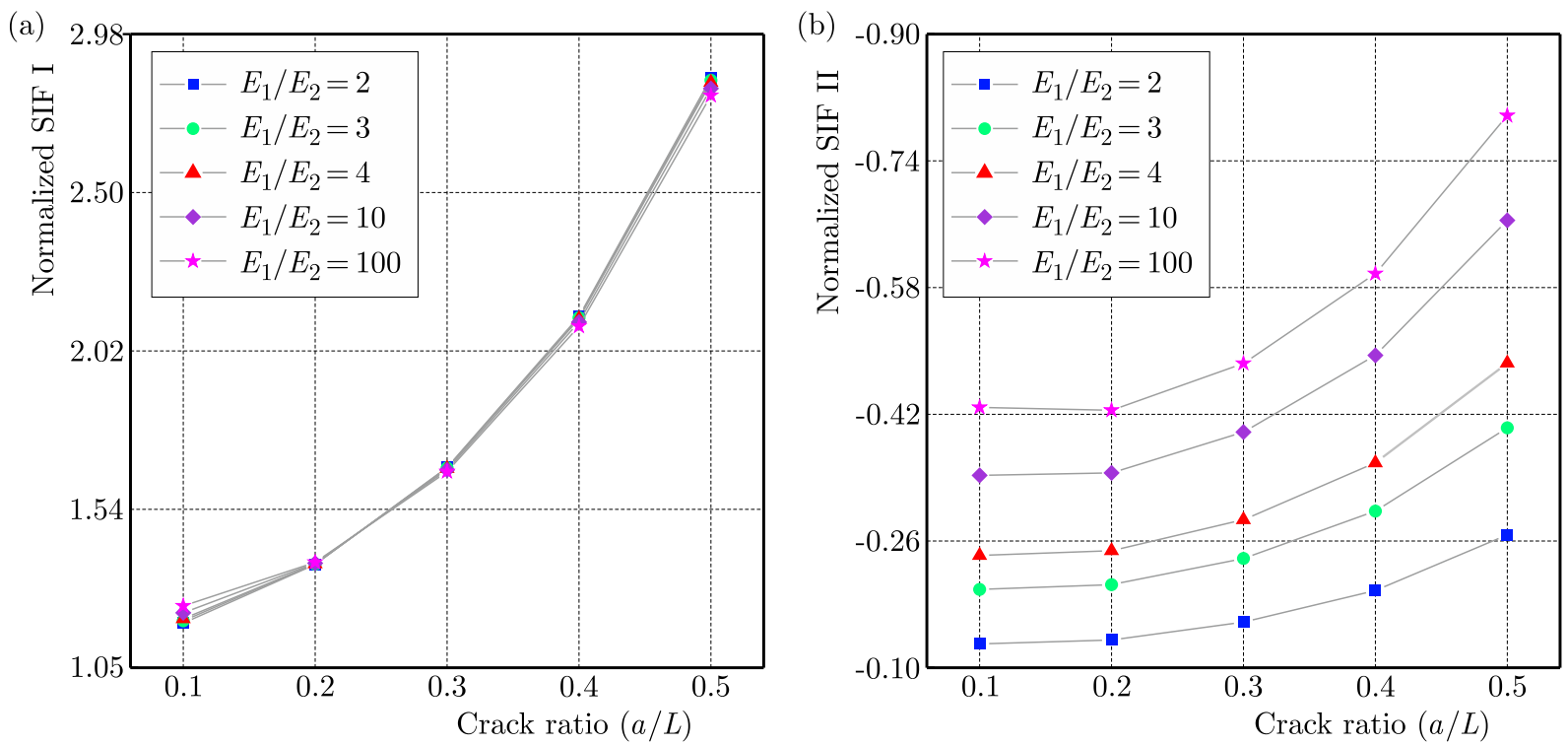

Fig. 14. The effect of Young's modulus ratio on the normalized SIF for the edge interface crack 
Table 3. Results of normalized stress intensity factors for the center interface crack

\begin{tabular}{|c|c|c|c|c|c|c|c|}
\hline \multirow{2}{*}{$E_{1} / E_{2}$} & \multirow{2}{*}{$2 a / L$} & \multicolumn{2}{|c|}{ Present study } & \multicolumn{2}{|c|}{ Matsumto et al. (2000) } & \multicolumn{2}{|c|}{ Miyazaki et al. (1993) } \\
\hline & & $\overline{K_{I}}$ & $\bar{K}_{I I}$ & $\bar{K}_{I}$ & $\bar{K}_{I I}$ & $\bar{K}_{I}$ & $\bar{K}_{I I}$ \\
\hline \multirow{5}{*}{2} & 0.1 & $\overline{c 1.006}$ & -0.0731 & $\overline{0.995}$ & $\begin{array}{c}-0.072 \\
\end{array}$ & $\overline{c 1.001}$ & $\begin{array}{c}-0.072 \\
\end{array}$ \\
\hline & 0.2 & 1.0245 & -0.0713 & 1.019 & -0.07 & 1.02 & -0.071 \\
\hline & 0.3 & 1.0572 & -0.071 & 1.053 & -0.072 & 1.053 & -0.071 \\
\hline & 0.4 & 1.1056 & -0.0725 & 1.104 & -0.073 & 1.104 & -0.073 \\
\hline & 0.5 & 1.1814 & -0.0764 & 1.18 & -0.077 & 1.181 & -0.077 \\
\hline \multirow{5}{*}{3} & 0.1 & 0.9993 & -0.1097 & 0.987 & -0.106 & 0.993 & -0.107 \\
\hline & 0.2 & 1.0179 & -0.1072 & 1.013 & -0.105 & 1.012 & -0.106 \\
\hline & 0.3 & 1.0504 & -0.1068 & 1.044 & -0.105 & 1.045 & -0.106 \\
\hline & 0.4 & 1.0981 & -0.1089 & 1.095 & -0.108 & 1.096 & -0.109 \\
\hline & 0.5 & 1.1726 & -0.1145 & 1.172 & -0.115 & 1.171 & -0.115 \\
\hline \multirow{5}{*}{4} & 0.1 & 0.9934 & -0.1314 & 0.981 & -0.128 & 0.987 & -0.129 \\
\hline & 0.2 & 1.0121 & -0.1284 & 1.006 & -0.126 & 1.006 & -0.127 \\
\hline & 0.3 & 1.0443 & -0.1279 & 1.037 & -0.126 & 1.031 & -0.127 \\
\hline & 0.4 & 1.0916 & -0.1303 & 1.088 & -0.131 & 1.089 & -0.13 \\
\hline & 0.5 & 1.1649 & -0.1368 & 1.163 & -0.136 & 1.163 & -0.137 \\
\hline \multirow{5}{*}{10} & 0.1 & 0.972 & -0.1764 & 0.962 & -0.172 & 0.968 & -0.174 \\
\hline & 0.2 & 0.9906 & -0.1729 & 0.987 & -0.168 & 0.986 & -0.171 \\
\hline & 0.3 & 1.0224 & -0.1708 & 1.017 & -0.171 & 1.018 & -0.17 \\
\hline & 0.4 & 1.0712 & -0.1745 & 1.065 & -0.172 & 1.066 & -0.173 \\
\hline & 0.5 & 1.1418 & -0.1838 & 1.135 & -0.181 & 1.136 & -0.182 \\
\hline \multirow{5}{*}{100} & 0.1 & 0.9488 & -0.2086 & 0.943 & -0.207 & 0.946 & -0.206 \\
\hline & 0.2 & 0.967 & -0.2043 & 0.964 & -0.201 & 0.964 & -0.201 \\
\hline & 0.3 & 0.9979 & -0.201 & 0.994 & -0.198 & 0.994 & -0.2 \\
\hline & 0.4 & 1.0435 & -0.204 & 1.039 & -0.2 & 1.039 & -0.203 \\
\hline & 0.5 & 1.1088 & -0.2129 & 1.104 & -0.208 & 1.104 & -0.21 \\
\hline
\end{tabular}

The results of the proposed method are closer to the BEM results than the XFEM results, as shown in Figs. 11 and 12. Young's modulus ratio has a slight effect on the SIFs, as shown in Tables 3 and 4. As it is obvious in the precedent examples, the local refinement property of analysis-suitable T-splines allows increasing the accuracy of the results and using less DOFs. Finally, we note that the evaluation of shape functions in XIGA is slower than some methods such as XFEM.

\section{Conclusion}

In this study, the analysis-suitable T-spline has been used in XIGA to approximate the solution in cracked bimaterial interfaces in order to construct geometry and to make local refinement around the discontinuities. Furthermore, it helps avoiding the emergence of superfluous control points during the local refinement process. The asymptotic crack-tip enrichment functions and the interaction integral method corresponding to bimaterial interface cracks have been used to evaluate the stress intensity factors. The results obtained by the proposed method have been compared with the results form literature, where a good agreement has been regarded demonstrating the accuracy of the approach. 
Table 4. Results of normalized stress intensity factors for the edge interface crack

\begin{tabular}{|c|c|c|c|c|c|c|c|c|c|}
\hline \multirow{2}{*}{$\frac{E_{1}}{E_{2}}$} & \multirow{2}{*}{$\frac{2 a}{L}$} & \multicolumn{2}{|c|}{ Present study } & \multicolumn{2}{|c|}{ Matsumto et al. (2000) } & \multicolumn{2}{|c|}{ Miyazaki et al. (1993) } & \multicolumn{2}{|c|}{ Liu et al. (2004) } \\
\hline & & $\overline{K_{I}}$ & $K K_{I I}$ & $\bar{K}_{I}$ & $\bar{K}_{I I}$ & $\bar{K}_{I}$ & $\bar{K}_{I I}$ & $\bar{K}_{I}$ & $\bar{K}_{I I}$ \\
\hline \multirow{5}{*}{2} & $\overline{\overline{0.1}}$ & 1.1899 & -0.1299 & 1.19 & -0.127 & 1.195 & -0.129 & - & - \\
\hline & 0.2 & 1.3682 & -0.1352 & 1.367 & -0.137 & 1.368 & -0.137 & 1.374 & -0.137 \\
\hline & 0.3 & 1.6619 & -0.1576 & 1.657 & -0.156 & 1.659 & -0.158 & 1.669 & -0.159 \\
\hline & 0.4 & 2.1198 & -0.1975 & 2.109 & -0.195 & 2.11 & -0.198 & 2.125 & -0.198 \\
\hline & 0.5 & 2.8423 & -0.2678 & 2.819 & -0.268 & 2.882 & -0.267 & 2.844 & -0.267 \\
\hline \multirow{5}{*}{3} & 0.1 & 1.1974 & -0.1988 & 1.198 & -0.195 & 1.203 & -0.197 & - & - \\
\hline & 0.2 & 1.369 & -0.2049 & 1.368 & -0.208 & 1.368 & -0.207 & 1.375 & -0.208 \\
\hline & 0.3 & 1.6603 & -0.2379 & 1.655 & -0.235 & 1.656 & -0.239 & 1.668 & -0.240 \\
\hline & 0.4 & 2.116 & -0.2977 & 2.102 & -0.298 & 2.105 & -0.298 & 2.121 & -0.299 \\
\hline & 0.5 & 2.8351 & -0.403 & 2.812 & -0.402 & 2.814 & -0.402 & 2.839 & -0.402 \\
\hline \multirow{5}{*}{10} & 0.1 & 1.2216 & -0.343 & 1.222 & -0.336 & 1.229 & -0.34 & - & - \\
\hline & 0.2 & 1.3719 & -0.3461 & 1.366 & -0.348 & 1.369 & -0.349 & 1.379 & -0.354 \\
\hline & 0.3 & 1.6547 & -0.3976 & 1.648 & -0.394 & 1.648 & -0.399 & 1.661 & -0.403 \\
\hline & 0.4 & 2.1023 & -0.4945 & 2.09 & -0.491 & 2.09 & -0.494 & 2.109 & -0.5 \\
\hline & 0.5 & 2.8103 & -0.6649 & 2.789 & -0.661 & 2.789 & -0.663 & 2.819 & -0.668 \\
\hline \multirow{5}{*}{100} & 0.1 & 1.2422 & -0.4286 & 1.251 & -0.424 & 1.251 & -0.424 & - & - \\
\hline & 0.2 & 1.3744 & -0.4252 & 1.376 & -0.429 & 1.370 & -0.428 & 1.381 & -0.434 \\
\hline & 0.3 & 1.6491 & -0.4842 & 1.647 & -0.47 & 1.642 & -0.485 & 1.657 & -0.494 \\
\hline & 0.4 & 2.0895 & -0.5975 & 2.083 & -0.569 & 2.078 & -0.597 & 2.101 & -0.608 \\
\hline & 0.5 & 2.7888 & -0.7972 & 2.772 & -0.793 & 2.77 & -0.797 & 2.804 & -0.813 \\
\hline
\end{tabular}

\section{References}

1. An X., Zhao Z., Zhang H., He L., 2013, Modeling bimaterial interface cracks using the numerical manifold method, Engineering Analysis with Boundary Elements, 37, 464-474

2. Anders D., Weinberg K., Reichardt R., 2012, Isogeometric analysis of thermal diffusion in binary blends, Computational Materials Science, 52, 182-188

3. Bazilevs Y., Akkerman I., 2010, Large eddy simulation of turbulent Taylor-Couette flow using isogeometric analysis and the residual-based variational multiscale method, Journal of Computational Physics, 229, 3402-3414

4. Bazilevs Y., Calo V., Zhang Y., Hughes T.J., 2006, Isogeometric fluid-structure interaction analysis with applications to arterial blood flow, Computational Mechanics, 38, 310-322

5. Belytschko T., Gracie R., 2007, On XFEM applications to dislocations and interfaces, International Journal of Plasticity, 23, 1721-1738

6. Benson D., Bazilevs Y., De Luycker E., Hsu M.C., Scott M., Hughes T., Belytschko, T., 2010, A generalized finite element formulation for arbitrary basis functions: from isogeometric analysis to XFEM, International Journal for Numerical Methods in Engineering, 83, 765-785

7. Bhardwaj, G., Singh, I., 2015, Fatigue crack growth analysis of a homogeneous plate in the presence of multiple defects using extended isogeometric analysis, Journal of the Brazilian Society of Mechanical Sciences and Engineering, 37, 1065-1082

8. Bhardwaj G., Singh I., Mishra B., 2015a, Fatigue crack growth in functionally graded material using homogenized XIGA, Composite Structures, 134, 269-284

9. Bhardwaj G., Singh I., Mishra B., 2015b, Stochastic fatigue crack growth simulation of interfacial crack in bi-layered FGMs using XIGA, Computer Methods in Applied Mechanics and Engineering, 284, 186-229 
10. Bhardwaj G., Singh I., Mishra B., Bui T., 2015c, Numerical simulation of functionally graded cracked plates using NURBS based XIGA under different loads and boundary conditions, Composite Structures, 126, 347-359

11. Borden M.J., Verhoosel C.V., Scott M.A., Hughes T.J., Landis C.M., 2012, A phase-field description of dynamic brittle fracture, Computer Methods in Applied Mechanics and Engineering, 217, 77-95

12. Buffa A., SAngalli G., VÁzQuez R., 2010, Isogeometric analysis in electromagnetics: B-splines approximation, Computer Methods in Applied Mechanics and Engineering, 199, 1143-1152

13. De Luycker, E., Benson D., Belytschko T., Bazilevs Y., Hsu M., 2011, X-FEM in isogeometric analysis for linear fracture mechanics, International Journal for Numerical Methods in Engineering, 87, 541-565

14. Erdogan F., 1963, Stress distribution in a nonhomogeneous elastic plane with cracks, Journal of Applied Mechanics, 30, 232-236

15. Evans J.A., Hughes T.J., 2013, Isogeometric divergence-conforming B-splines for the steady Navier-Stokes equations, Mathematical Models and Methods in Applied Sciences, 23, 1421-1478

16. Evans A.G., Rühle M., Dalgleish B.J., Charalambides P.G., 1990, The fracture energy of bimaterial interfaces, Metallurgical Transactions A, 21, 2419-2429

17. Ghorashi S.S., Mohammadi S., Sabbagh-Yazdi, S.-R., 2011, Orthotropic enriched element free Galerkin method for fracture analysis of composites, Engineering Fracture Mechanics, 78, 1906-1927

18. Ghorashi S.S., Valizadeh N., Mohammadi S., 2012, Extended isogeometric analysis for simulation of stationary and propagating cracks, International Journal for Numerical Methods in Engineering, 89, 1069-1101

19. Ghorashi S.S., Valizadeh N., Mohammadi S., Rabczuk T., 2015, T-spline based XIGA for fracture analysis of orthotropic media, Composite Structures, 147, 138-146

20. Hsu M.-C., Akkerman I., Bazilevs Y., 2011, High-performance computing of wind turbine aerodynamics using isogeometric analysis, Computers and Fluids, 49, 93-100

21. Hughes T.J., Cottrell J.A., Bazilevs Y., 2005, Isogeometric analysis: CAD, finite elements, NURBS, exact geometry and mesh refinement, Computer Methods in Applied Mechanics and Engineering, 194, 4135-4195

22. Hutchinson J.W., Mear M., Rice J.R., 1987, Crack paralleling an interface between dissimilar materials, Journal of Applied Mechanics, 54, 828-832

23. Ikeda T., Nagai M., Yamanaga K., Mryazaki N., 2006, Stress intensity factor analyses of interface cracks between dissimilar anisotropic materials using the finite element method, Engineering Fracture Mechanics, 73, 2067-2079

24. Jia Y., Anitescu C., Ghorashi S.S., Rabczuk T., 2015, Extended isogeometric analysis for material interface problems, IMA Journal of Applied Mathematics, 80, 608-633

25. Kalali A.T., Hassani B., Hadidi-Moud S., 2016, Elastic-plastic analysis of pressure vessels and rotating disks made of functionally graded materials using the isogeometric approach, Journal of Theoretical and Applied Mechanics, 54, 113-125

26. Lee K.Y., Choi H.J., 1988, Boundary element analysis of stress intensity factors for bimaterial interface cracks, Engineering Fracture Mechanics, 29, 461-472

27. Li X., Zheng J., Sederberg T.W., Hughes T.J., Scott M.A., 2012, On linear independence of T-spline blending functions, Computer Aided Geometric Design, 29, 63-76

28. Liu X., Xiao Q.Z., Karihaloo B., 2004, XFEM for direct evaluation of mixed mode SIFs in homogeneous and bi-materials, International Journal for Numerical Methods in Engineering, 59, 1103-1118 
29. Matsumto T., Tanaka M., Obara R., 2000, Computation of stress intensity factors of interface cracks based on interaction energy release rates and BEM sensitivity analysis, Engineering Fracture Mechanics, 65, 683-702

30. Miyazaki N., Ikeda T., Soda T., Munakata T., 1993, Stress intensity factor analysis of interface crack using boundary element methodapplication of contour-integral method, Engineering Fracture Mechanics, 45, 599-610

31. Mö̈s N., Cloirec M., Cartraud P., Remacle J.-F., 2003, A computational approach to handle complex microstructure geometries, Computer Methods in Applied Mechanics and Engineering, 192, 3163-3177

32. Nagashima T., Омото Y., Tani S., 2003, Stress intensity factor analysis of interface cracks using X-FEM, International Journal for Numerical Methods in Engineering, 56, 1151-1173

33. Nguyen V.P., Anitescu C., Bordas, S.P., Rabczuk T., 2015, Isogeometric analysis: An overview and computer implementation aspects, Mathematics and Computers in Simulation, 117, 89-116

34. Nguyen V.P., Kerfriden P., Bordas S.P., 2014, Two-and three-dimensional isogeometric cohesive elements for composite delamination analysis, Composites Part B: Engineering, 60, 193212

35. NGuyen V.P., Nguyen-Xuan H., 2013, High-order B-splines based finite elements for delamination analysis of laminated composites, Composite Structures, 102, 261-275

36. Pant M., Singh I., Mishra B., 2011, Evaluation of mixed mode stress intensity factors for interface cracks using EFGM, Applied Mathematical Modelling, 35, 3443-3459

37. Peković O., Stupar S., Simonović A., Svorcan J., Trivković S., 2015, Free vibration and buckling analysis of higher order laminated composite plates using the isogeometric approach, Journal of Theoretical and Applied Mechanics, 53, 453-466

38. Peng X., Atroshchenko E., Simpson R., Kulasegaram S., Bordas S., 2014, Crack growth analysis by a NURBS-based isogeometric boundary element method, 11th World Congress on Computational Mechanics, Barcelona, Spain

39. Rice J., 1988, Elastic fracture mechanics concepts for interfacial cracks, Journal of Applied Mechanics, 55, 98-103

40. Rice J., Sin G.C., 1965, Plane problems of cracks in dissimilar media, Journal of Applied Mechanics, 32, 418-423

41. Scott M., Li X., Sederberg T., Hughes T., 2012, Local refinement of analysis-suitable Tsplines, Computer Methods in Applied Mechanics and Engineering, 213, 206-222

42. Sederberg T.W., Cardon D.L., Finnigan G.T., North N.S., Zheng J., Lyche T., 2004, T-spline simplification and local refinement, ACM Transactions on Graphics (TOG), 23, 276-283

43. Sederberg T.W., Zheng J., Bakenov A., Nasri A., 2003, T-splines and T-NURCCs, ACM Transactions on Graphics (TOG), 22, 477-484

44. Sukumar N., Huang Z., Prévost J.H., Suo Z., 2004, Partition of unity enrichment for bimaterial interface cracks, International Journal for Numerical Methods in Engineering, 59, 1075-1102

45. Sun C.T., Jin C., 1987, On strain energy release rates for interfacial cracks in bi-material media, Engineering Fracture Mechanics, 28, 13-20

46. TAda H., Paris P., Irwin G., 2000, The Analysis of Cracks Handbook, ASME Press 2, 1, New York, USA

47. Temizer I., Wriggers P., Hughes T., 2011, Contact treatment in isogeometric analysis with NURBS, Computer Methods in Applied Mechanics and Engineering, 200, 1100-1112

48. Verhoosel C.V., Scott M.A., De Borst R., Hughes T.J., 2011, An isogeometric approach to cohesive zone modeling, International Journal for Numerical Methods in Engineering, 87, 336-360 
49. Williams M., 1959, The stresses around a fault or crack in dissimilar media, Bulletin of the Seismological Society of America, 49, 199-204

50. YAU J., WANG S., 1984, An analysis of interface cracks between dissimilar isotropic materials using conservation integrals in elasticity, Engineering Fracture Mechanics, 20, 423-432

51. YuUki R., Xu J.-Q., 1994, Boundary element analysis of dissimilar materials and interface crack, Computational Mechanics, 14, 116-127

52. Zhou Z., Xu X., Leung A.Y., Huang Y., 2013, Stress intensity factors and T-stress for an edge interface crack by symplectic expansion, Engineering Fracture Mechanics, 102, 334-347

53. Zhou Z.H., Leung A.Y.T., Xu X.S., 2014, The finite element discretized symplectic method for interface cracks, Composites Part B: Engineering, 58, 335-342

Manuscript received March 7, 2016; accepted for print May 5, 2016 